\title{
An ultra-accurate numerical method in the design of liquid phononic crystals with hard inclusion
}

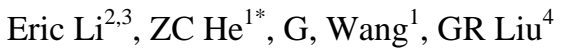 \\ ${ }^{I}$ State Key Laboratory of Advanced Design and Manufacturing for Vehicle Body, Hunan University, \\ Changsha, 410082 P. R. China \\ ${ }^{2}$ Department of Mechanical and Automation Engineering, The Chinese University of Hong Kong, Shatin, \\ NT, Hong Kong, China \\ ${ }^{3}$ The State Key Laboratory of Fluid Power and Mechatronic Systems, Zhejiang University, Hangzhou, \\ 310027, China \\ ${ }^{4}$ Department of Aerospace Engineering and Engineering Mechanics, University of Cincinnati, \\ Cincinnati, OH 45221-0070, USA
}

\begin{abstract}
The phonocis crystals (PCs) are periodic man-made composite materials. In this paper, a mass-redistributed finite element method (MR-FEM) is formulated to study the wave propagation within liquid PCs with hard inclusion. With a perfect balance between stiffness and mass in the MR-FEM model, the dispersion error of longitudinal wave is minimized by redistribution of mass. Such tuning can be easily achieved by adjusting the parameter $r$ that controls the location of integration points of mass matrix. More importantly, the property of mass conservation in the MR-FEM model indicates that the locations of integration points inside or outside the element are immaterial. Four numerical examples are studied in this work, including liquid PCs with cross and circle hard inclusions, different size of inclusion and defect. Compared with standard finite element method (FEM), the numerical results have verified the accuracy and effectiveness of MR-FEM. The proposed MR-FEM is a unique and innovative numerical approach with its outstanding features, which has strong potentials to study the stress wave within multi-physics PCs.
\end{abstract}

Key words: Liquid phononic crystals; MR-FEM; band gap; Numerical method

\footnotetext{
${ }^{1 *}$ State Key Laboratory of Advanced Design and Manufacturing for Vehicle Body, Hunan University, Changsha, 410082 P. R. China' Email address: hezhicheng815@hnu.edu.cn
} 


\section{Introduction}

Recently, the manipulation and control of elastic or acoustic waves in periodic composite materials such as phononics crystals (PCs) have attracted considerable attention. The periodic PCs have many novel and unique properties that do not exist in nature, which can be tailored by changing the configuration and microscopic size of the individual constituents instead of chemical components. One of the most attractive features of PCs is the appearance of frequency band gaps, which is able to prohibit the propagation of elastic or acoustic wave within PCs. The remarkable progress in the field of band gap of PCs with combination of 3D printing technology has led to many useful devices such as cavities, lens, switches, acoustic waveguides and filters [1-3].

There are two types of working mechanism to create the band gap of PCs, which are known as Bragg scattering and locally resonant. Unfortunately, it is almost impossible to obtain Bragg band gaps in the low frequency range as the wavelength of the elastic waves must be in the same order of the dimension of microstructure based on the Bragg scattering mechanism [4], which has become a big hindrance in the practical application of PCs to shield the low frequency wave [5]. To this end, Liu et al. [6] developed the locally resonant sonic materials (LRSMs) in 2000, which is composed of periodic lead balls immersed in the epoxy matrix. The experimental study indicated that the lattice constant of LRSMs could be much smaller compared with the longitudinal wavelength within epoxy at the low frequency gap. Consequently, a large amount of research efforts on theoretical analysis of PCs has been explored. In 2004, Wang et al. discovered locally resonance PCs with binary structure [7]. Lin and Huang proposed a 2D PCs with various anisotropic cylinders in an isotropic host, leading to tunable band gap. [8]. Hussein and 
Frazier analyzed a formal treatment of the Bloch wave propagation problem for viscously damped PCs [9]. Recently, Hussein et al. have provided an overview of phononic materials and structures [10]. In general, we can define fluid-fluid, solid-solid, and mixed solid-fluid composite PCs [11]. In this study, we limit ourselves to liquid PCs with hard inclusion.

On the other hand, the development of efficient numerical methods is very significant to design PCs to satisfy the specific requirements. In the earlier stage, the plane-wave expansion (PWE) method was often applied to study the wave propagation in PCs $[12,13]$. Recently, Plane wave expansion and variational techniques were combined to compute the band gap of phononic crystals (PCs) $[14,15]$. However, the PWE has some inherent problems to analyze the media with a huge difference of elastic properties [16]. In order to overcome this difficulty, Finite-difference time-domain (FDTD) has been applied to investigate the mechanism of band gap in the PCs [17]. Although the implementation of FDTD is quite straightforward, it is very difficult to solve the problems with complex geometries. As a reliable, robust and effective technique, finite element method (FEM) is widely employed to study the acoustic and elastic waves in periodic PCs and other types of composite materials due to its versatility to deal with complex geometry. Wang et al. proposed a lumped-mass method with faster convergence rate to analyze the propagations of elastic wave in two dimensional PCs [16]. Liu and Gao developed an explicit dynamic FEM to compute the band-structure of 2D PCs [18]. Furthermore, the XFEM was formulated to compute the band structure of 3D mechanical metamaterials with complicated geometry [19]. 
However, the standard FEM still has some drawbacks to model the propagation of longitudinal wave in the liquid PCs due to its overly-stiff property [20]. It is well known the numerical dispersion error of standard FEM model will deteriorate in higher frequency range [21]. Based on the rule of thumb, FEM fails to provide very accurate solutions unless the mesh is sufficiently fine. Although the use of more elements per wavelength is a possible way to improve the accuracy of longitudinal wave problems, the use of fine mesh will cause the dramatic increase of computational cost. Such issue could be worse for large scale 3D PCs problems with iterative process.

In fact, the accuracy of the numerical solutions in the simulation of acoustic wave based on our past research is strongly related to the correct balance of the mass and stiffness [22-26]. In order to improve the FEM results, one effective way is to soften the over-stiff of stiffness created by FEM. Recently, by introducing the gradient smoothing operation, Liu and his group conducted intensive research on the softness of FEM using triangular and tetrahedral elements, and various types of smoothed finite element methods (S-FEM) [27-30] in the frame of meshfree and FEM were developed. The SFEM with a proper softened effect can produce more accurate solutions compared with standard FEM [21, 26, 31-35].

The alternative way to reduce the dispersion error in the propagation of longitudinal wave is to tune the balance between the mass and stiffness, which is termed as massredistributed finite element method (MR-FEM) [24, 36, 37]. In the present MR-FEM, the stiffness matrix is created by standard FEM, but the mass matrix of the discretization system is formulated by introducing three flexible integration points with a parameter $r(r$ controls the locations of integration points of mass matrix). The numerical results have 
clearly demonstrated that the present MR-FEM method works ideally in the simulation of the propagation of longitudinal wave in periodic PCs with hard inclusion. The numerical results from MR-FEM model are more accurate compared with standard FEM using consistent or lumped mass matrices. Furthermore, the physical meanings and numerical implications for mass conservation in the framework of MR-FEM are discussed in detail. The novel property of mass conservation ensures that MR-FEM formulated in the model of liquid PCs is able to use more general integration points, even outside the elements, which is very significant to study the linear/nonlinear longitudinal wave.

The aim of this paper is to formulate an efficient and accurate MR-FEM to improve the computational efficiency in the modeling and simulation of longitudinal wave propagation within the liquid PCs with hard inclusion, and the rest of the paper is structured as follows. In Section 2, the detailed formulation of MR-FEM is presented for the band structure analysis of liquid PCs. The discussion to minimize the dispersion error of longitudinal wave in the liquid PCs is detailed in Section 3 and Appendix. In Section 4, four numerical examples are presented to verify the effectiveness of the proposed 2D MR-FEM. The conclusions are then made in Section 5.

\section{Theory and numerical method}

\subsection{Equation of wave propagation in the liquid PCs}

It is well known that only longitudinal wave exists in periodic composite media of liquid or gas host, and the governing equation of longitudinal wave in such isotropic media is expressed as follows:

$$
\Delta p+\zeta^{2} p=0
$$


where $\Delta$ stands for the Laplace operator, $p$ caused by longitudinal wave is the spatial distribution of complex acoustic pressure, $\zeta$ is the wavenumber defined by

$$
\zeta=\frac{\omega}{c}
$$

where $\omega$ is the angular frequency, $c$ represents the speed of sound traveling in the fluid. Based on the Bloch theorem, the acoustic pressure can be expressed in the following form:

$$
\mathbf{p}(\mathbf{r})=e^{i(\mathbf{k} \cdot \mathbf{r})} \mathbf{p}_{\mathbf{k}}(\mathbf{r})
$$

where $i=\sqrt{-1}, \mathbf{k}=\left(k_{x}, k_{y}\right)$ is the wave vector, and $\mathbf{p}_{\mathbf{k}}(\mathbf{r})$ is a periodic vector function with the same periodicity crystal lattice.

The discretized form of the eigenvalue equations in the unit cell can be written as

$$
\left(\mathbf{K}-\omega^{2} \mathbf{M}\right) \mathbf{p}=0
$$

where

$$
\begin{aligned}
& \mathbf{K}=\int_{\Omega} \nabla \boldsymbol{\psi}^{\mathrm{T}} \nabla \boldsymbol{\psi} \mathrm{d} \Omega \\
& \mathbf{M}=\rho \int_{\Omega} \boldsymbol{\psi}^{\mathrm{T}} \boldsymbol{\psi} \mathrm{d} \Omega
\end{aligned}
$$

where $\boldsymbol{\psi}$ is the shape function matrix, and $\rho$ is the density of fluid.

As shown in Fig. 1, the Bloch theorem is imposed on the boundaries of the unit cell in the direction where the periodicity applies. Thus, the following relation between the pressure $\mathbf{P}(\mathbf{r})$ for the nodes on the boundary of the unit cell is given as follows[38]:

$$
\mathbf{p}(\mathbf{r}+\mathbf{a})=e^{i(\mathbf{k} \cdot \mathbf{a})} \mathbf{p}(\mathbf{r})
$$

where a is the lattice constant. By introducing the periodic boundary condition, the Eq.(5) 
can be further written to the following reduced equation:

$$
\left[\mathbf{K}\left(k_{x}, k_{y}\right)-\omega^{2} \mathbf{M}\left(k_{x}, k_{y}\right)\right] \mathbf{p}=0
$$

The eigenfrequencies of the above equation can be solved for any given each pair of $\left(k_{x}, k_{y}\right)$

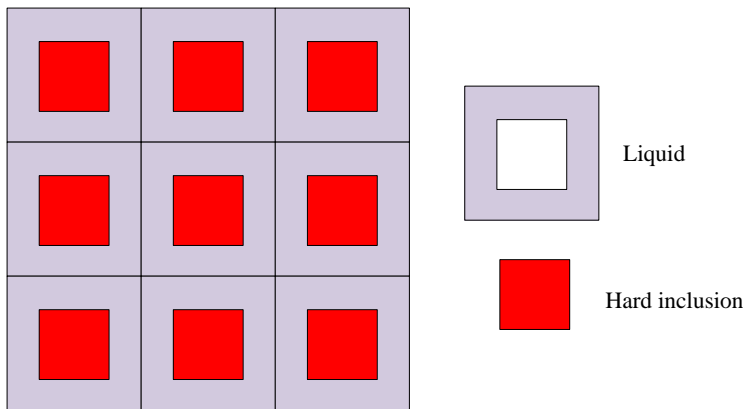

a) Geometric representation of the two-dimensional periodic systems of hard rods in liquid

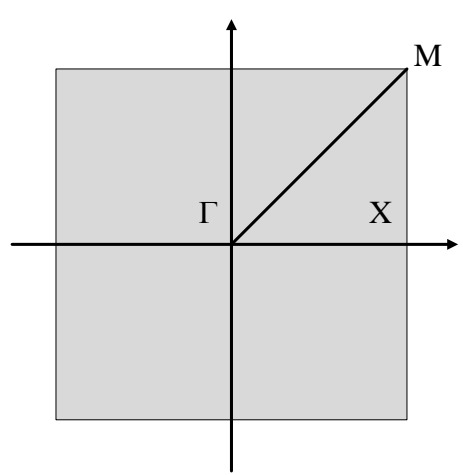

b) First Brillouinzone

Figure 1: Liquid PCs embedded with hard inclusion

\subsection{Formulation of MR-FEM}

In the integration of stiffness and mass (Eqs. (5) and (6)), it is very straightforward to transform the physical element to parent element as shown in Fig. 2:

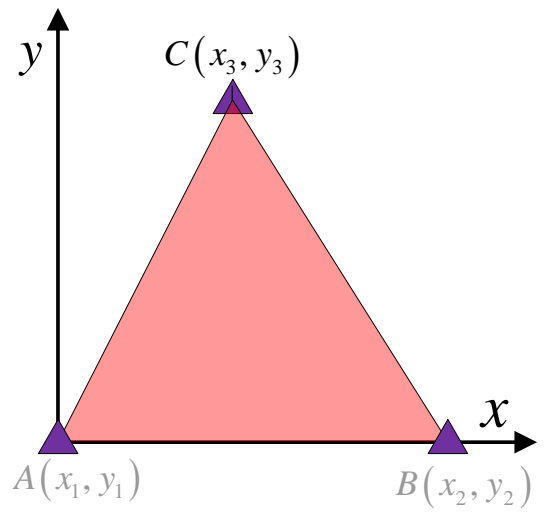

a) Physical element

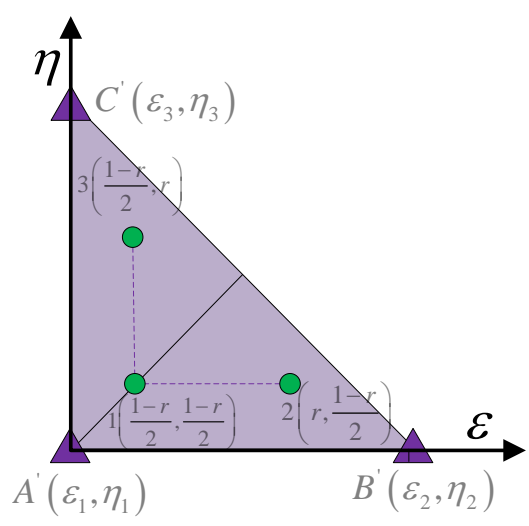

b) Parent element

Nodes of field

Integration point

Figure 2: Flexible integration points 


$$
\begin{gathered}
\mathbf{K}=\int_{-1}^{+1} \int_{-1}^{+1}(\nabla \boldsymbol{\psi})^{\mathrm{T}}(\nabla \boldsymbol{\psi}) \operatorname{det}(J) \mathrm{d} \xi \mathrm{d} \eta \\
\mathbf{M}=\int_{-1}^{+1} \int_{-1}^{+1} \boldsymbol{\psi}^{\mathrm{T}} \boldsymbol{\psi} \operatorname{det}(J) \mathrm{d} \xi \mathrm{d} \eta
\end{gathered}
$$

where $J$ is Jacobian matrix, and $\xi, \eta$ are local coordinates. The shape functions $\psi$ are needed to satisfy the Partitions of Unity (PU) [39]:

$$
\sum_{i \in S_{x}} \psi_{i}(\mathbf{x})=1
$$

where $S_{x}$ is the set of the nodes in the local support domain containing $\mathbf{x}$.

As the derivative of the shape function using linear triangular elements $\nabla \boldsymbol{\psi}$ is constant, the integration of stiffness is the integrand multiplied by the area of the element domain. The integration of mass matrix is obtained by the conventional Gauss integral method [36]:

$$
\rho \int_{\Omega^{e}} \boldsymbol{\psi}^{\mathrm{T}} \boldsymbol{\psi} \mathrm{d} \Omega=\rho A^{e} \sum_{I=1}^{n g}\left[w_{I}\left(\boldsymbol{\psi}^{\mathrm{T}} \boldsymbol{\psi}\right)_{\text {evaluated at point } i}\right]
$$

where $I$ stands for the $I$ th sampling point of the numerical integration, $w_{I}$ are the weights (the weights $w_{I}=1 / 3$ are adopted for all the three sampling integration points), $A_{\mathrm{e}}$ represents the area of the triangular element, and $n g$ is the number of Gauss points applied to integration. In the MR-FEM using linear triangular elements, three flexible points are adopted to evaluate the mass matrix as shown in Fig. 2(b), which are expressed as follows:

$$
\text { Point 1: }\left(\frac{1-r}{2} \frac{1-r}{2}\right) ; \text { Point 2: }\left(r \frac{1-r}{2}\right) ; \text { Point 3: }\left(\begin{array}{cc}
\frac{1-r}{2} & r
\end{array}\right)
$$

where $r$ is the parameter that controls the locations of integration points.

As $r=1$ is corresponding to the following three integration points, the MR-FEM is the 
same as the lumped mass matrix:

Point 1: $(0,0)$; Point 2: $(1,0)$; Point 3: $(0,1)$

As $r=0$ and $r=2 / 3$ are corresponding to the following three integration points, the MR-FEM becomes the consistent mass matrix:

$$
r=0 \rightarrow \text { Point 1: }(1 / 2,1 / 2) ; \text { Point 2: }(0,1 / 2) ; \text { Point 3: }(1 / 2,0)
$$

$r=2 / 3 \rightarrow$ Point $1:(1 / 6,1 / 6) ;$ Point 2: $(2 / 3,1 / 6) ;$ Point $3:(2 / 3,1 / 6)$

By using three flexible integration points expressed in Eq. (13), the element mass matrix using linear triangular element can be derived with a parameter $r$ :

$$
\mathbf{M}^{\mathrm{e}}=\frac{1}{3} \rho A^{e}\left[\begin{array}{ccc}
r^{2}+\frac{(1-r)^{2}}{2} & r(1-r)+\frac{(1-r)^{2}}{4} & r(1-r)+\frac{(1-r)^{2}}{4} \\
r(1-r)+\frac{(1-r)^{2}}{4} & r^{2}+\frac{(1-r)^{2}}{2} & r(1-r)+\frac{(1-r)^{2}}{4} \\
r(1-r)+\frac{(1-r)^{2}}{4} & r(1-r)+\frac{(1-r)^{2}}{4} & r^{2}+\frac{(1-r)^{2}}{2}
\end{array}\right]
$$

It is noted that that the set of nodal shape functions has the property of the partitions of unity (PU). At the same time, the summation of the weights used for all the integral points is equal to $1\left(\sum_{i \in S_{x}} \psi_{i}(\mathbf{x})=1\right)$. These are the sufficient conditions to guarantee the mass conservation at element level [39].

$$
\begin{aligned}
m_{e} & =\rho \iint_{A_{e}} \sum_{i=1}^{n_{e}} \sum_{j=1}^{n_{e}} m_{i j} \mathrm{~d} A=\rho \iint_{A_{e}} \sum_{i=1}^{n_{e}} \sum_{j=1}^{n_{e}} \psi_{i}(\mathbf{x}) \psi_{j}(\mathbf{x}) \mathrm{d} A \\
& =\rho \iint_{A_{e}} \underbrace{\sum_{i=1}^{n_{e}} \psi_{i}(\mathbf{x})}_{P U=1} \underbrace{\sum_{j=1}^{n_{e}} \psi_{j}(\mathbf{x}) \mathrm{d} A}_{P U=1} \\
& =\rho \iint_{A_{e}} \mathrm{~d} A=\rho A_{e}
\end{aligned}
$$




\section{Minimization of dispersion error of longitudinal wave}

In our previous work, the minimization of dispersion error of acoustic wave is strictly inside the element. This implies $0 \leq r \leq 1$. However, the mathematical proof of mass conservation enables us to use more flexible values, even the integration point outside the element as shown in Fig. 3. In this section, the theoretical analysis to use the integration point outside the element $(r<0$ or $r>1)$ is discussed.

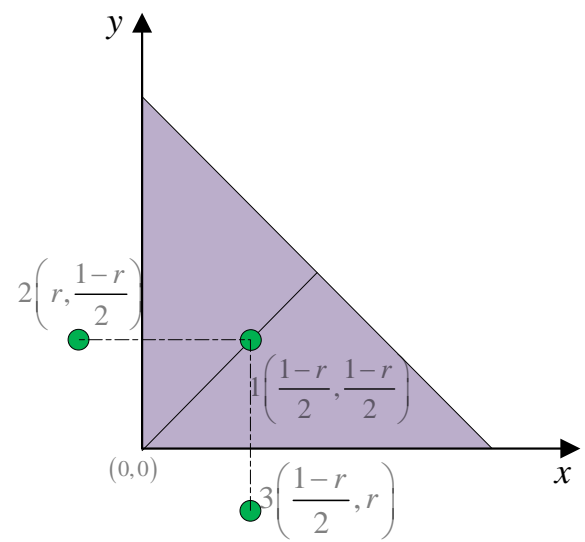

Integration point

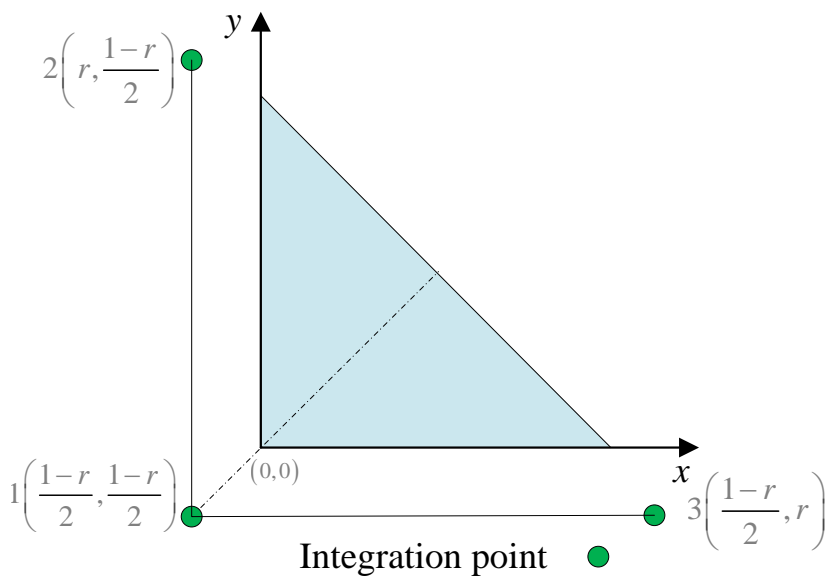

b) $r>1$

a) $r<0$

Figure 3: Integration points outside the triangular element

Based on the dispersion error analysis conducted in Appendix, the optimal value of $r$

that controls the locations of integration point is: $r=\frac{4 \pm \sqrt{16+4 \times 6}}{12}=\frac{2 \pm \sqrt{10}}{6}$.

The value of $r \in[0,1]$ ensures the locations of integration points inside the triangular element. As the PU property has both necessary and sufficient conditions to ensure the mass conservation for wave propagation problems, it gives us more freedom to tune the balance between the stiffness and mass. The following equation reveals some important details based on numerical integration technique. 


$$
\begin{aligned}
m_{e} & =\iint_{A_{e}} \sum_{i=1}^{n_{e}} \sum_{j=1}^{n_{e}} \psi_{i}(\mathbf{x}) \psi_{j}(\mathbf{x}) d A=\sum_{I}^{n_{g}} \rho w_{I}\left(\sum_{i=1}^{n_{e}} \sum_{j=1}^{n_{e}} \psi_{i}\left(\mathbf{x}_{I}\right) \psi_{j}\left(\mathbf{x}_{I}\right)\right) \\
& =\sum_{I}^{n_{g}} \rho w_{I}(\underbrace{\sum_{i=1}^{n_{e}} \psi_{i}\left(\mathbf{x}_{I}\right)}_{\because P U=1, \therefore=1} \underbrace{\sum_{j=1}^{n_{e}} \psi_{j}\left(\mathbf{x}_{I}\right)}_{\because P U=1, \therefore=1})=\rho \sum_{I}^{n_{g}} w_{I}
\end{aligned}
$$

Eq. (18) obviously indicates that the mass conservation is always satisfied at the element level [39] as long as the sum of the weights is equal to the area of the element. Such important conclusion using numerical integration approach is also valid for any subdomain in an element. This provides a sound proof that we do not always require the location of integration point inside the element in the framework of MR-FEM.

As the location of the sampling points used on the numerical integration of mass matrix is immaterial in terms of the mass conservation, the optimal $r$ value can be either $r=0.8604$ or $r=-0.1937$ to achieve the perfect balance between the stiffness and mass in the analysis of the propagation of linear longitudinal wave.

Next, we further examine the property of eigenvalue based on the general formulation of mass matrix. By using single triangular element, the eigenvalues of the discretized system can be expressed as follows:

$$
\varsigma_{1}=0 ; \varsigma_{2}=\frac{6}{A^{e}\left(9 r^{2}-6 r+1\right)} ; \varsigma_{3}=\frac{18}{A^{e}\left(9 r^{2}-6 r+1\right)}
$$

It is seen that the eigenvalues are strongly related to the term $9 r^{2}-6 r+1$. The relationship between $r$ and the term $9 r^{2}-6 r+1$ is shown in Fig. 4. For more convenient discussion, the range of $r$ between $[-1,2]$ is analyzed. The domain of $r$ value between [0, 1] represents the location inside the element. The domains of $r<0$ or $r>1$ stand for the locations outside the triangular element. We can see that the eigenvalues are symmetrical 
with $r=1 / 3$. As shown in Fig. 4, it is found that the parameter $r$ has a great effect on the eigenvalue. As $r>1 / 3$, the eigenvalue is inversely proportional to the parameter $r$. On the other hand, the eigenvalue increases with decreasing the parameter $r$ as $r<1 / 3$.

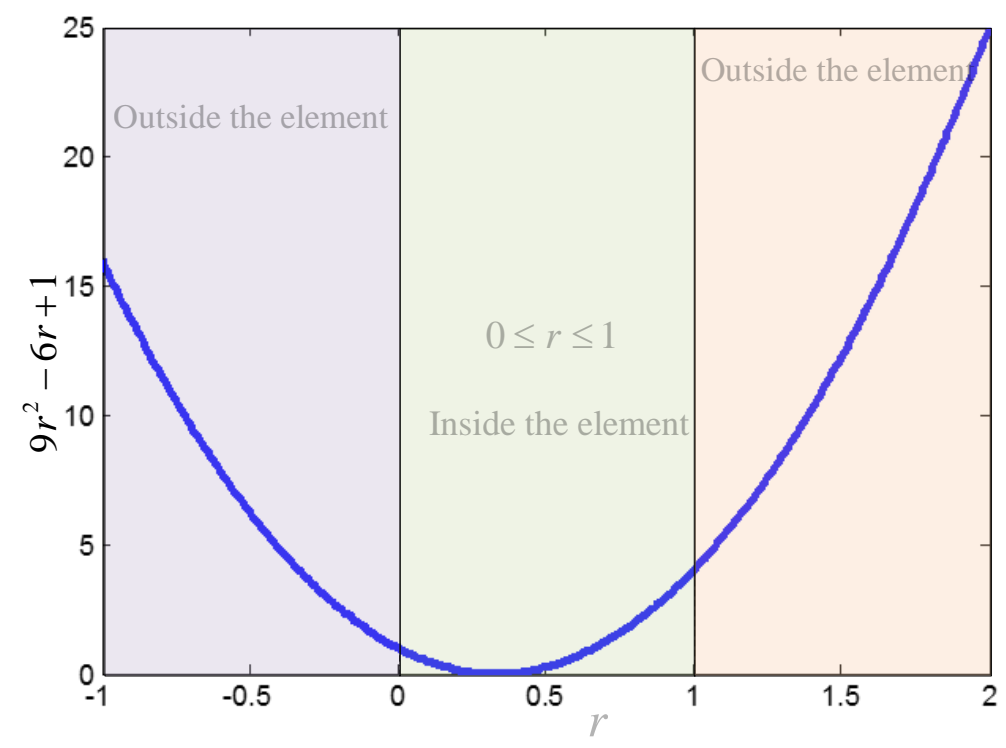

Figure 4: Effect of integration point on the eigenvalue $(-1 \leq r \leq 2)$

In this work, the optimal integration point $r=0.8604$ or $r=-0.1937$ is only valid for the isotropic fluid. In the cases of anisotropic and nonlinear fluid, the novel property of mass conservation in the MR-FEM enables us to tune the balance between the stiffness and mass using the flexible parameter $r$ without restriction of location. In this regards, to obtain a perfect balance system in the nonlinear model, the conventional location of integration points strictly inside the element (or boundary of the element) does not need to hold. Such optimal value $r$ that controls the locations of integration points can be derived in the same way of dispersion error analysis. In addition, the development of such perfect balance systems can be extended to elastic wave in Multiphysics PCs domain. Such interesting and important research can be considered in our future work. 


\section{Numerical examples and discussion}

In this section, four examples are analyzed to demonstrate the excellent advantages of the present MR-FEM using flexible integration points (inside or outside of the element). In order to study the numerical results quantitatively, the error indicator of eigenfrequency along $\mathrm{M}, \Gamma, \mathrm{X}$ directions is defined as:

$$
\text { Error }=\sum_{i=1}^{n} \sqrt{\frac{\left(\varsigma_{i}^{\text {num }}-\varsigma_{i}^{\text {exact }}\right)^{2}}{\left(\varsigma_{i}^{\text {exact }}\right)^{2}}} / n
$$

where $\varsigma_{\text {num }}$ stands for the eigenfrequency from MR-FEM and FEM, and $\varsigma_{\text {exact }}$ represents the exact eigenfrequency or the reference solution using very fine mesh. In these four examples, the hard inclusion is immersed with the water. The material properties for water are taken as follows: $\rho_{f}=1000 \mathrm{~kg} / \mathrm{m}^{3}$, and the speed of longitudinal wave in the water is $1500 \mathrm{~m} / \mathrm{s}$.

\subsection{Liquid PCs with cross section}

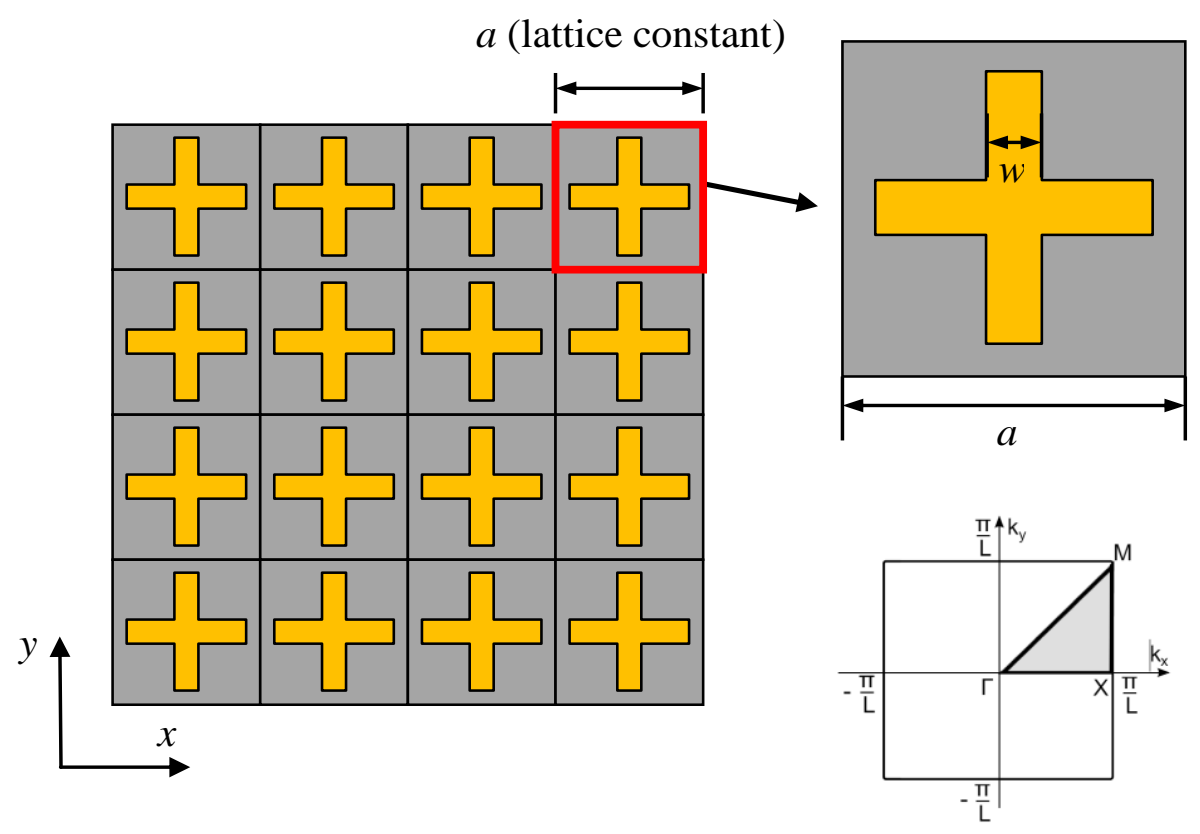

Figure 5: Liquid PCs with a cross section of hard inclusion 
First, a square PCs structure consisting of a cross section of hard inclusion is studied, as shown in Fig. 5. The lattice constant is $a=0.15 \mathrm{~m}$, and the length and width of hard cross section are $l=0.12 \mathrm{~m}, w=0.03 \mathrm{~m}$ respectively. Fig. 6 presents the band gap computed by FEM (consistent and lumped mass) and MR-FEM using the same type of mesh (135 nodes, 198 triangular elements). In order to verify the numerical results obtained from MR-FEM, the reference results obtained from commercial software COMSOL using very mesh (8480 nodes) are plotted together. At the lower frequency, it is noticed that all numerical solutions agree very well with the reference solutions. However, the dispersion errors in the standard FEM model (consistent and lumped mass) become larger at higher frequency. In contrast to the FEM model, it is clearly seen that the numerical solutions from MR-FEM using coarse mesh have an excellent agreement with the reference results even at high frequency.

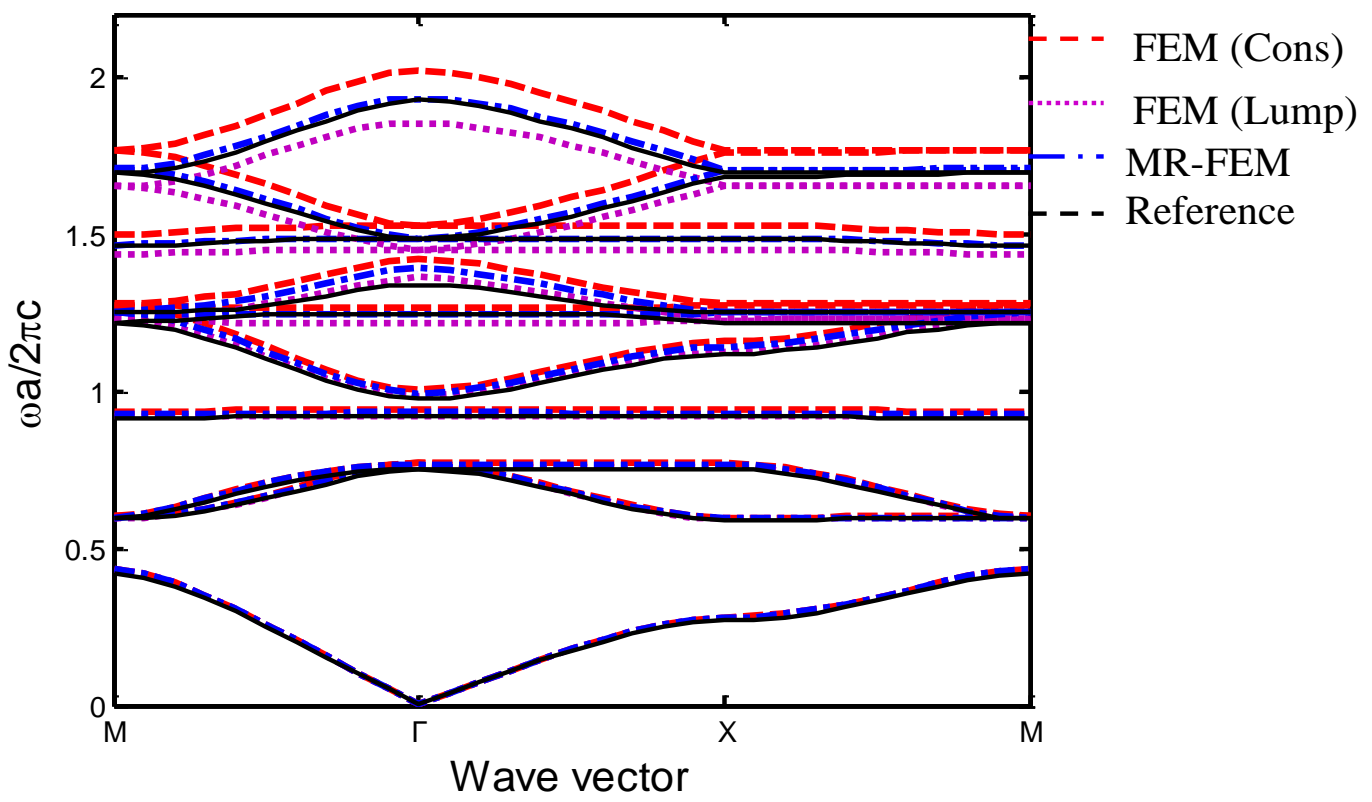

Figure 6: Comparison of band gap 
The properties of convergence rate of FEM (consistent and lumped mass) and MRFEM models for the first twenty eigenfrequencies (along $M, \Gamma, X$ directions) are further studied. Using Eq. (20), the error of eigenfrequencies is calculated and plotted against the average nodal spacing $(h)$ as shown in Fig. 7. Four types of set of mesh using linear triangular elements, as shown in Table 1, are employed in this study. It is obviously noticed that MR-FEM always gives the best solutions compared with standard FEM, which has strongly demonstrated the outstanding features of MR-FEM.

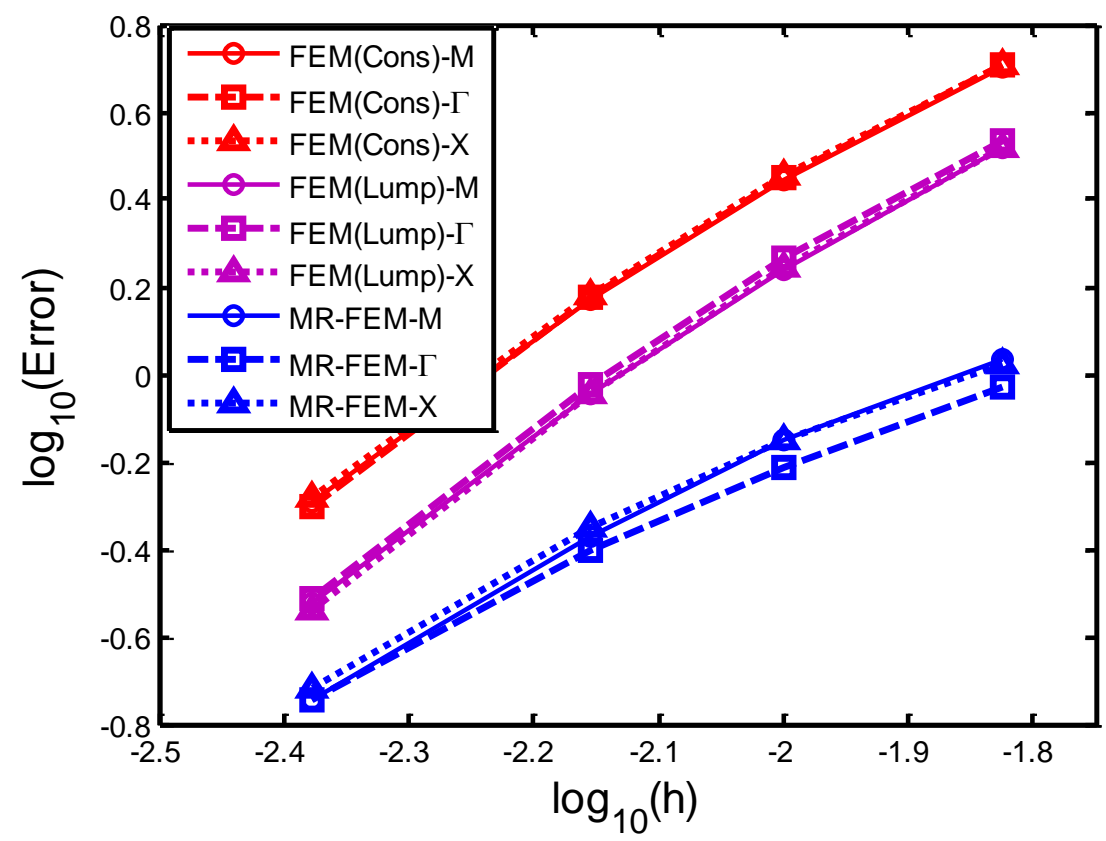

Figure 7: The convergence rate of the first twenty eigenfrequencies

Table 1: Four sets of mesh

\begin{tabular}{cccc}
\hline \hline Model & Size of element & Number of node & Number of element \\
1 & 0.015 & 135 & 198 \\
2 & 0.010 & 234 & 356 \\
3 & 0.071 & 423 & 698 \\
4 & 0.0042 & 1179 & 2098 \\
\hline \hline
\end{tabular}

The computational efficiency of MR-FEM and FEM (consistent and lumped mass matrices) is studied in Fig. 8. With the same number of node, it is seen that the 
computational time for FEM and MR-FEM is almost identical. However, with consideration of computational time versus accuracy, the MR-FEM shows very significant advantages compared with FEM using consistent and lumped mass matrices.

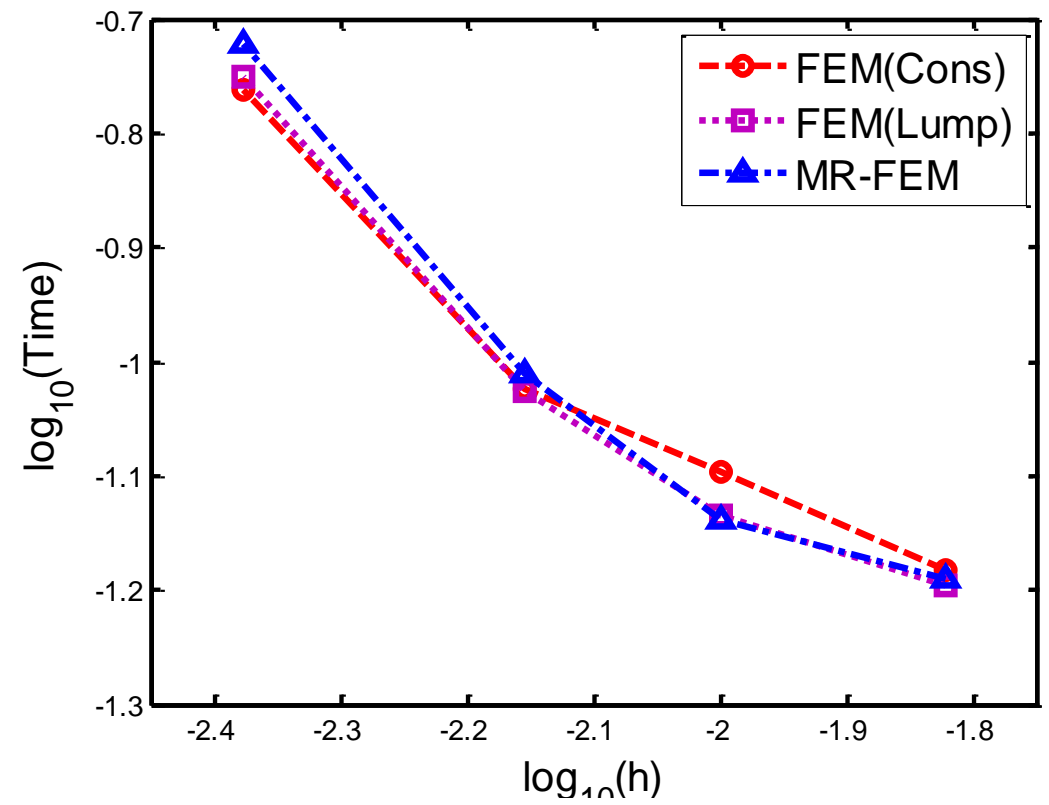

(a) Computational time

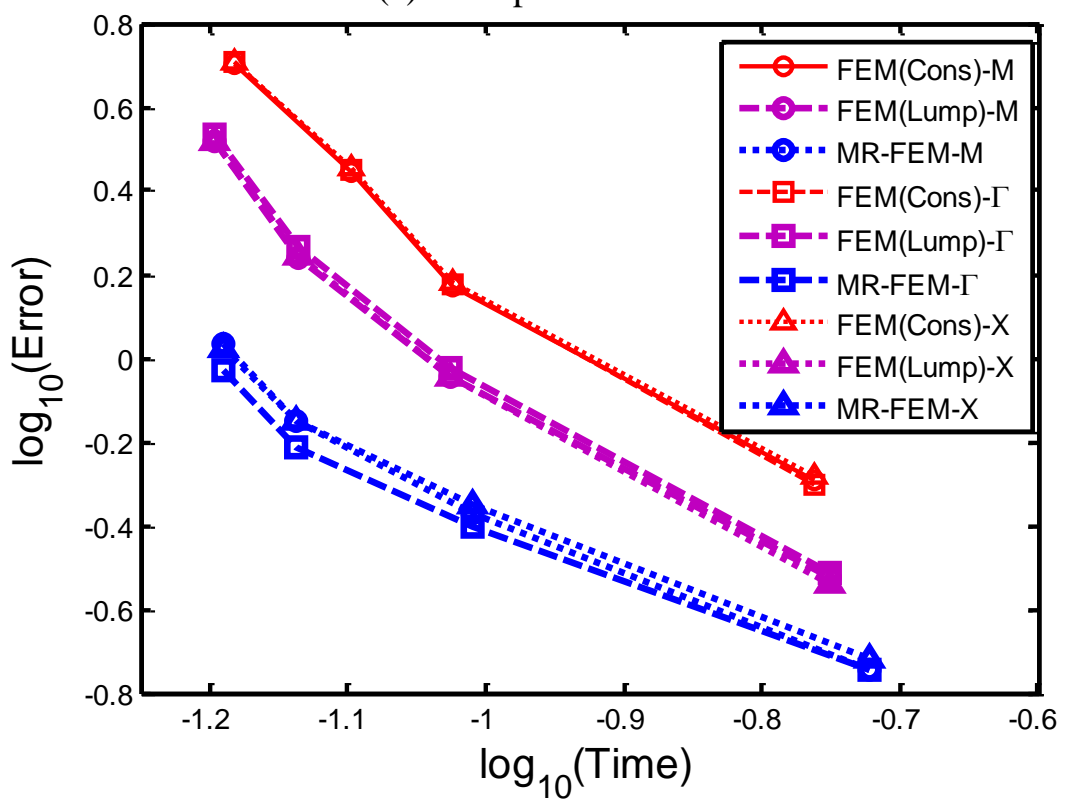

(b) Computational efficiency

Figure 8: Comparison of computational efficiency

Next, the pressure response at finite liquid PCs is studied. As outlined in Fig. 9, the unit pressure is applied to the left hand side of the $4 \times 4$ cells in horizontal direction. The 
frequency response is obtained at the center of its right hand side. The reference solutions are obtained from COMSOL using very fine mesh (9055 nodes, 16396 triangular elements). As expected, the numerical results from MR-FEM model are much better than those using FEM with consistent and lumped mass matrices with the same coarse mesh (750 nodes, 1130 linear triangular elements) as outlined in Fig. 10. It is noted that there exist several eigenmodes in the vicinity of the $4000 \mathrm{~Hz}$. In the vicinity of eigenfrequencies, the problem becomes numerically ill-posed, which significantly increases the numerical error [40]. Therefore, the errors between coarse mesh (FEM and MR-FEM models) and fine mesh (reference model) become larger. In addition, in order to ensure accurate solutions, the relationship between the size of element and frequency in the MR-FEM model should follow "the rule of thumb" [41].

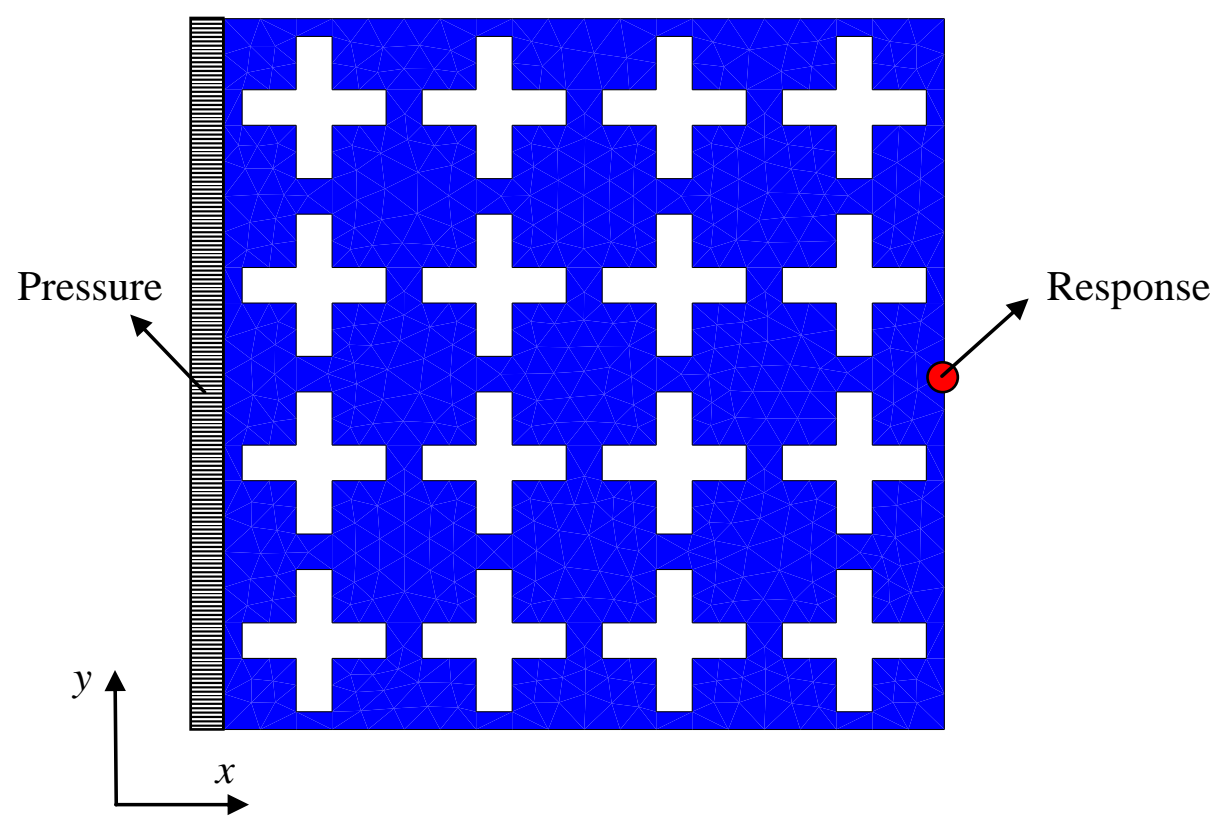

Figure 9: $4 \times 4$ unit cell 


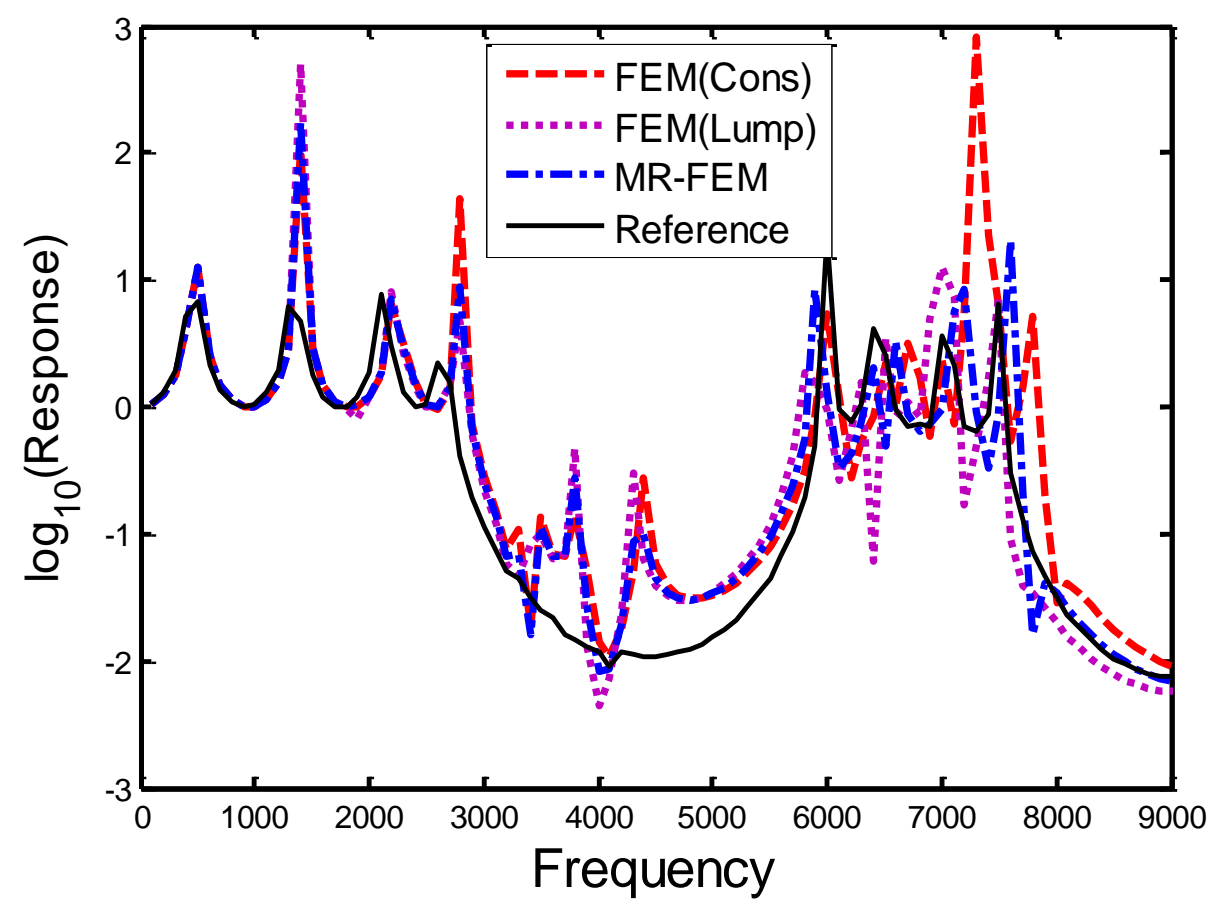

Figure 10: Pressure response using various numerical methods

From this example, it is seen that two optimal values ( $r=0.8604$ and $r=-0.1937)$ in the MR-FEM has achieved the same numerical results. That has verified the property of the mass conservation from numerical perspective. Again, the optimal $r$ value that controls the locations of integration points results in a perfect balance system, which is irrelevant to mesh size and material properties. That is extremely useful to design liquid PCs with excellent computational efficiency. It is noted that these two optimal values are only valid for the propagation of linear longitudinal wave. However, we can always tune the balance between the stiffness and mass matrices using flexible integration points based on the dispersion error. 


\subsection{Liquid PCs with a circular section}

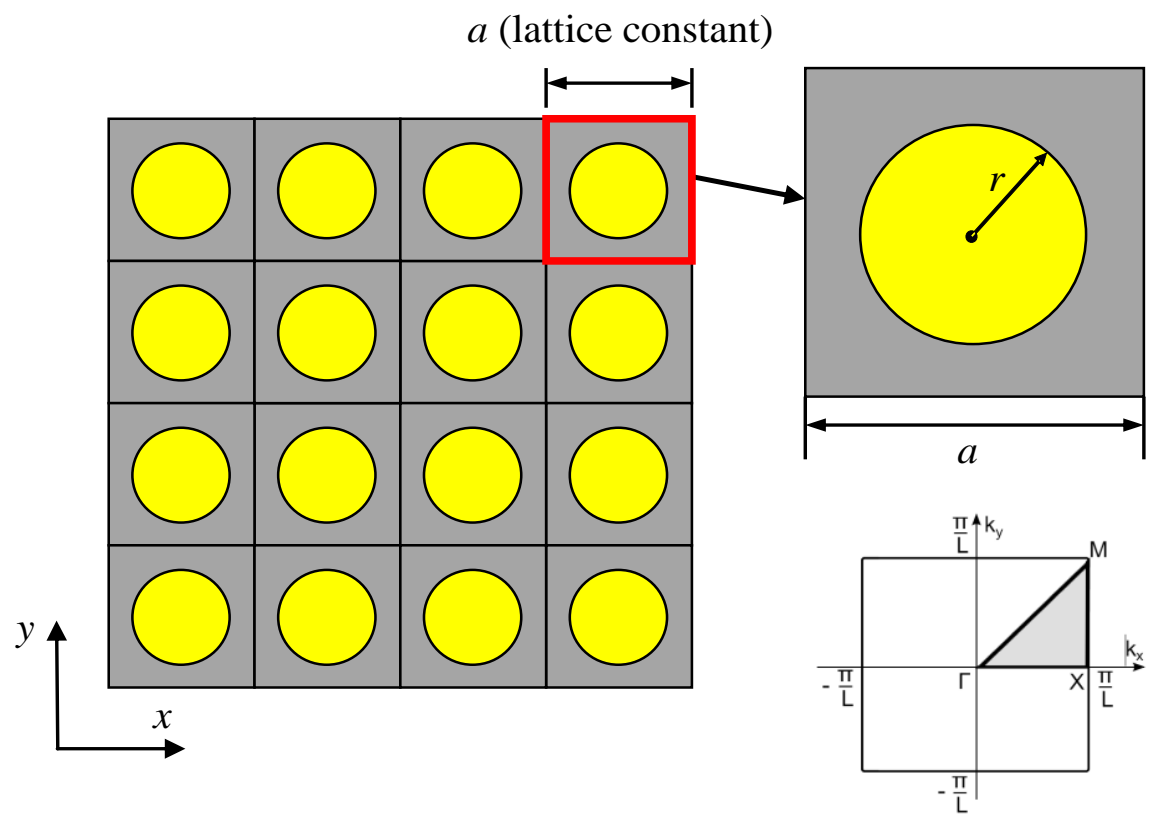

Figure 11: Liquid PCs with a circular section of hard inclusion

The second example is a square liquid PCs with circular section of hard inclusion as outlined in Fig. 11. In this example, lattice constant $a=0.15 \mathrm{~m}$, and the radius of hard inclusion is $r_{i n}=0.05 \mathrm{~m}$. The computed band gap using FEM and MR-FEM with same type of coarse mesh (135 nodes, 198 triangular element) is presented in Fig. 12. The reference solutions are obtained from COMSOL using very fine mesh (15621 nodes, 30328 triangular elements). At the lower frequency, the numerical results from FEM and MR-FEM have an excellent agreement with the reference solutions. However, there is a large deviation between FEM results (both consistent and lumped mass matrices) and reference model. This is due to the imbalance of stiffness and mass in the FEM model. On the contrary, the MR-FEM with a perfect balance system always provides very accurate solutions from lower to higher frequency. 


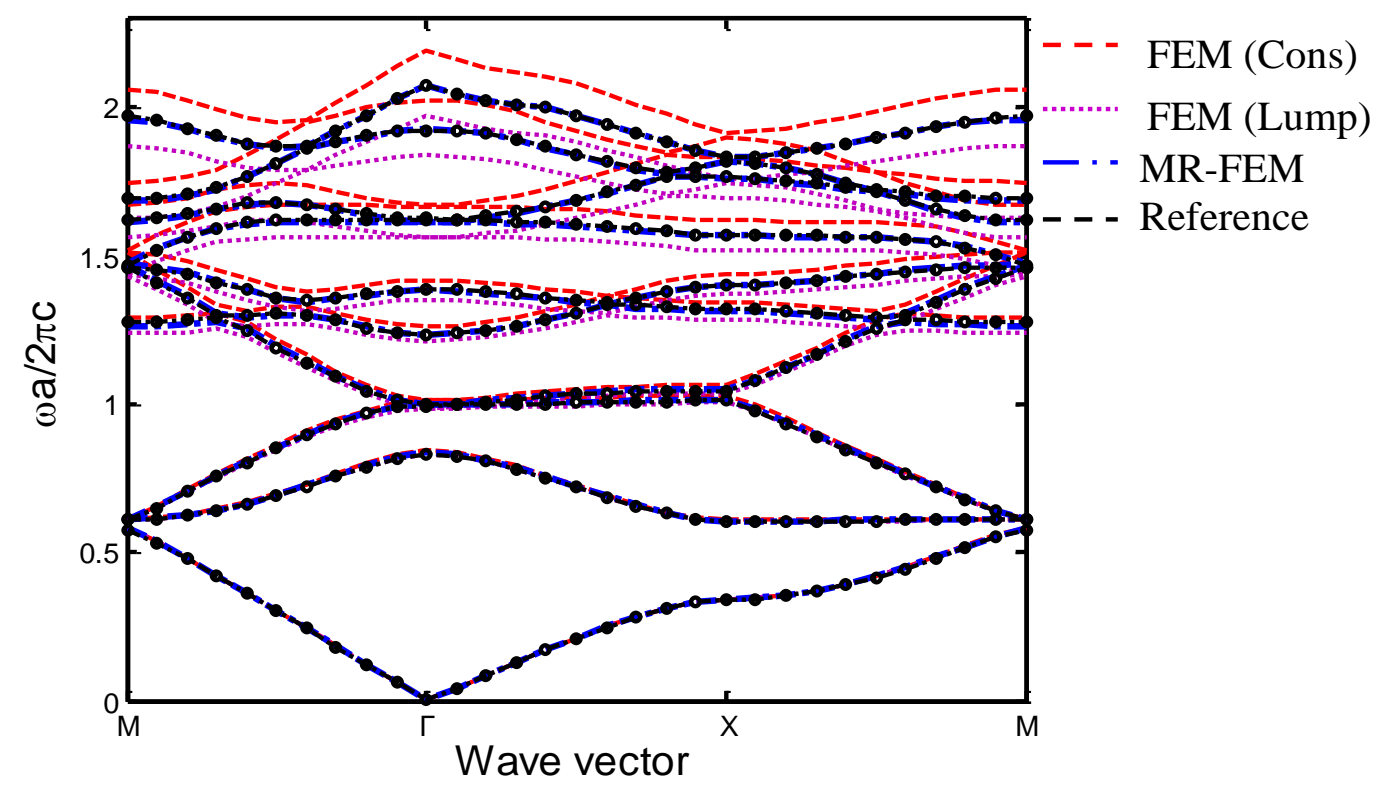

Figure 12: The computed band gap from various numerical methods

The convergence rates of MR-FEM and FEM are analyzed for the first twenty eigenfrequencies in Fig. 13. Four sets of mesh using linear triangular elements as shown in Table 2 are used in this study. It is seen that the accuracy of FEM from consistent and lumped mass matrices is almost the same. Again, the MR-FEM shows very excellent accuracy among all the numerical methods as depicted in Fig. 13.

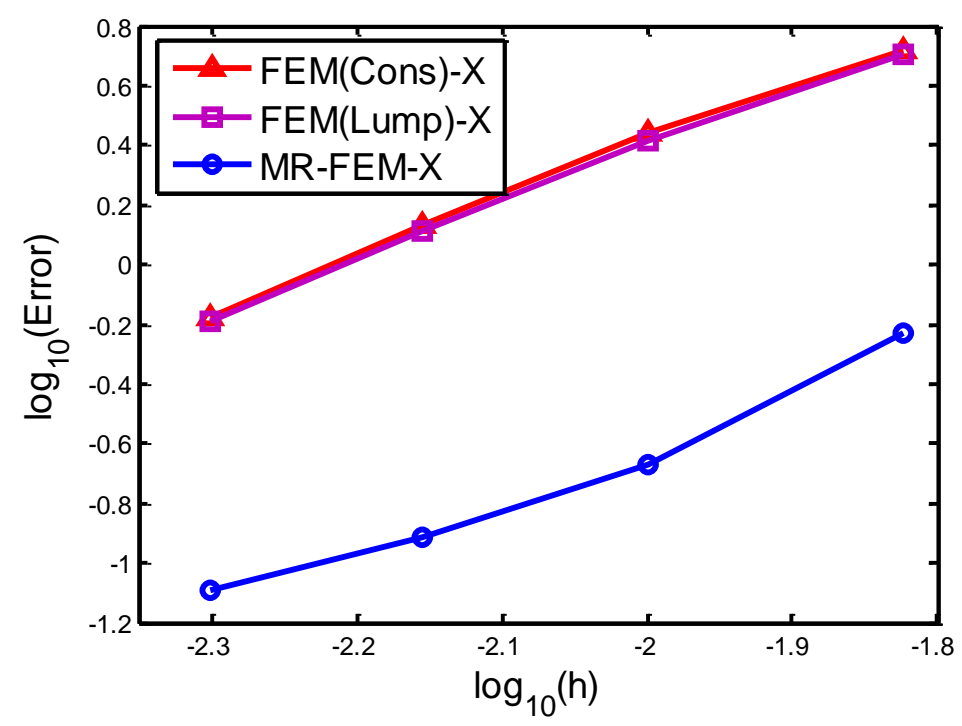

Figure 13: The convergence rates for the first twenty eigenfrequencies 
Table 2: Four sets of mesh

\begin{tabular}{cccc}
\hline \hline Model & Size of element & Number of node & Number of element \\
1 & 0.015 & 109 & 157 \\
2 & 0.010 & 194 & 296 \\
3 & 0.0073 & 360 & 591 \\
4 & 0.005 & 696 & 1209 \\
\hline \hline
\end{tabular}

The performance of computational efficiency using FEM and MR-FEM is further examined in Fig. 14. It is seen that with the same set of meshes, the computational time for FEM and MR-FEM is almost the same as shown in Fig. 14(a). However, after taking the results accuracy into account and considering the efficiency, the present MR-FEM is found to perform much better than the FEM using lumped and consistent mass matrices for the results of eigenfrequency as shown in Fig. 14(b).

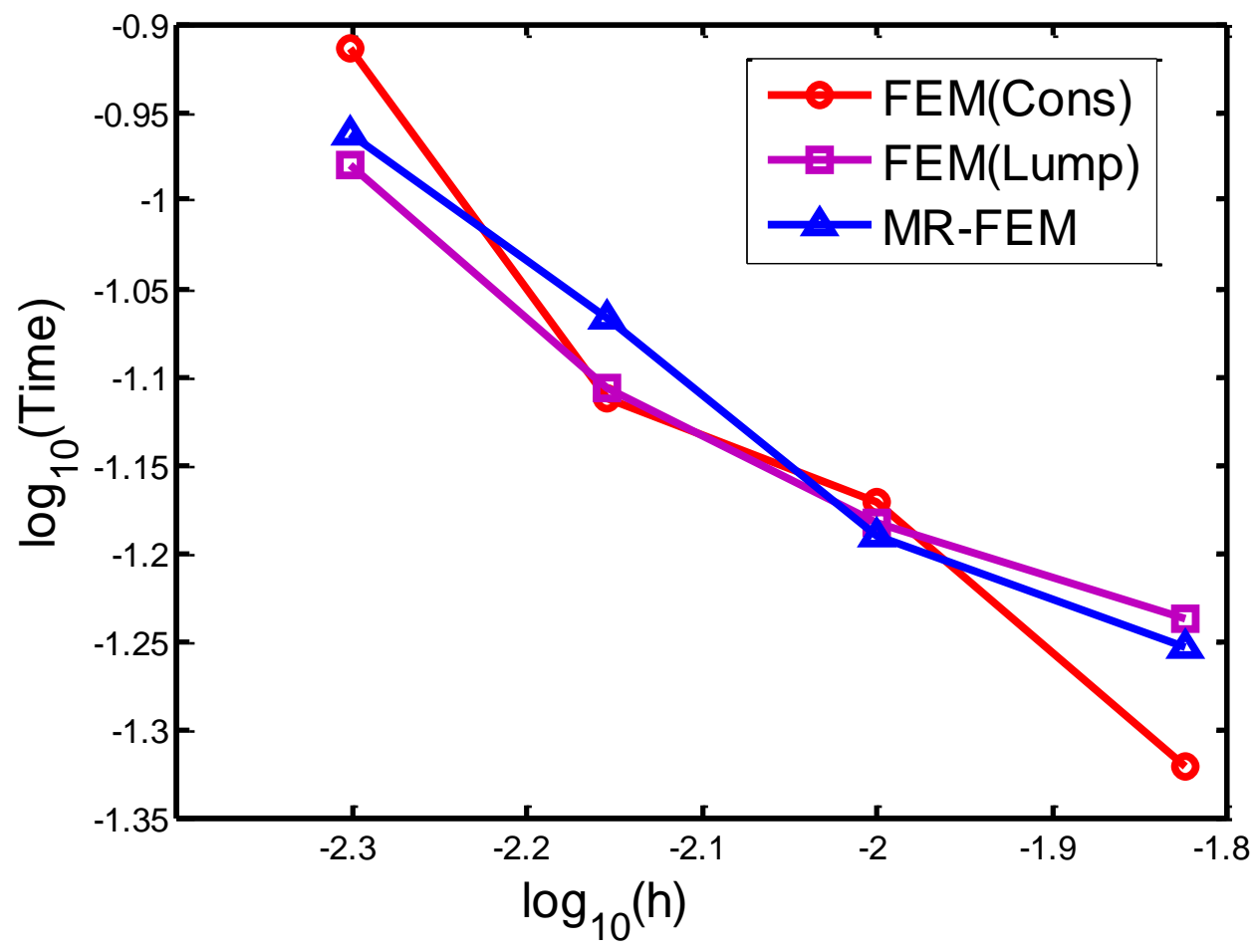

(a) Computational time 


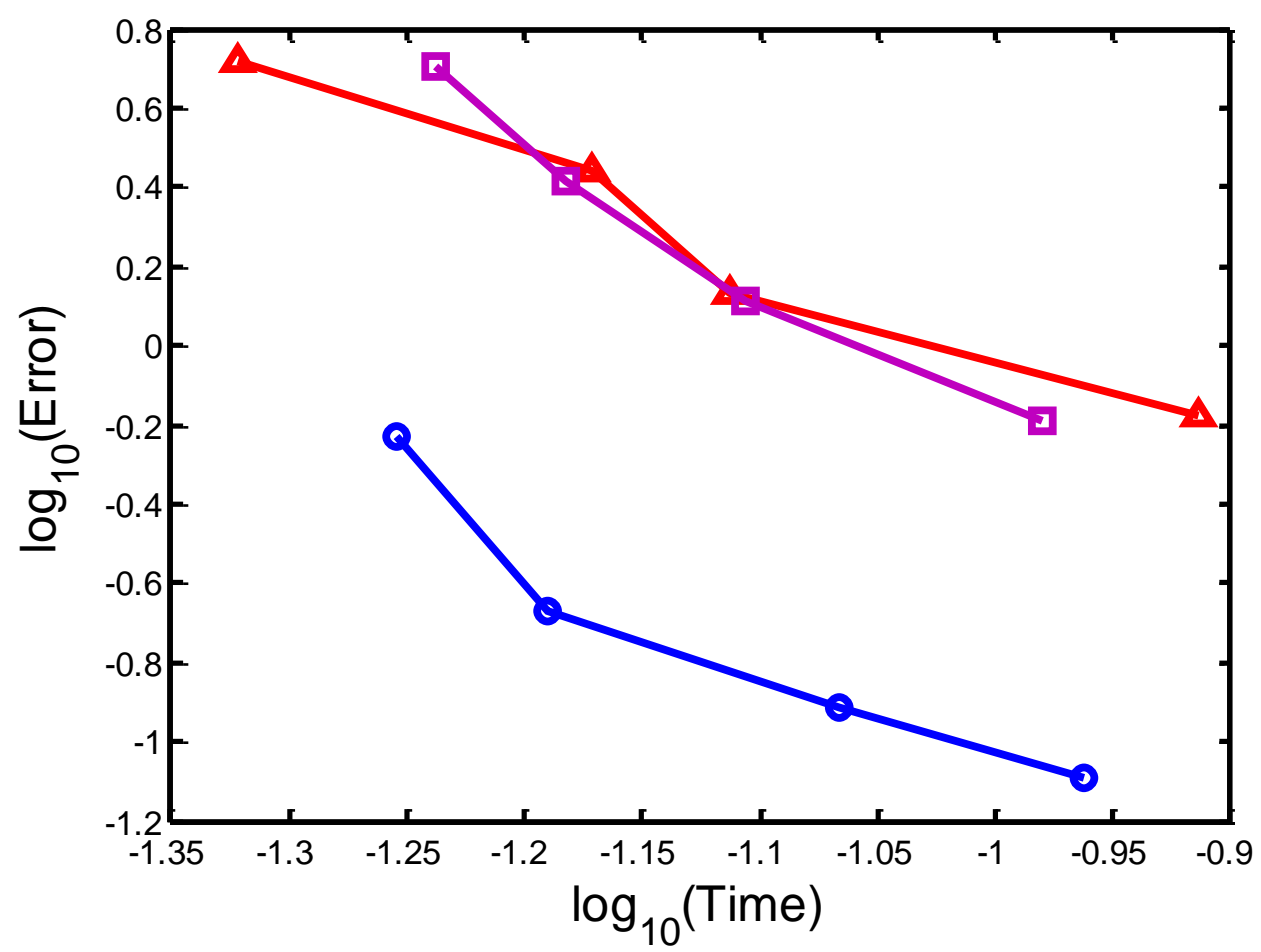

(b) Computational efficiency

Figure 14: Comparison of computational efficiency

\subsection{Liquid PCs with different size of hard inclusion}

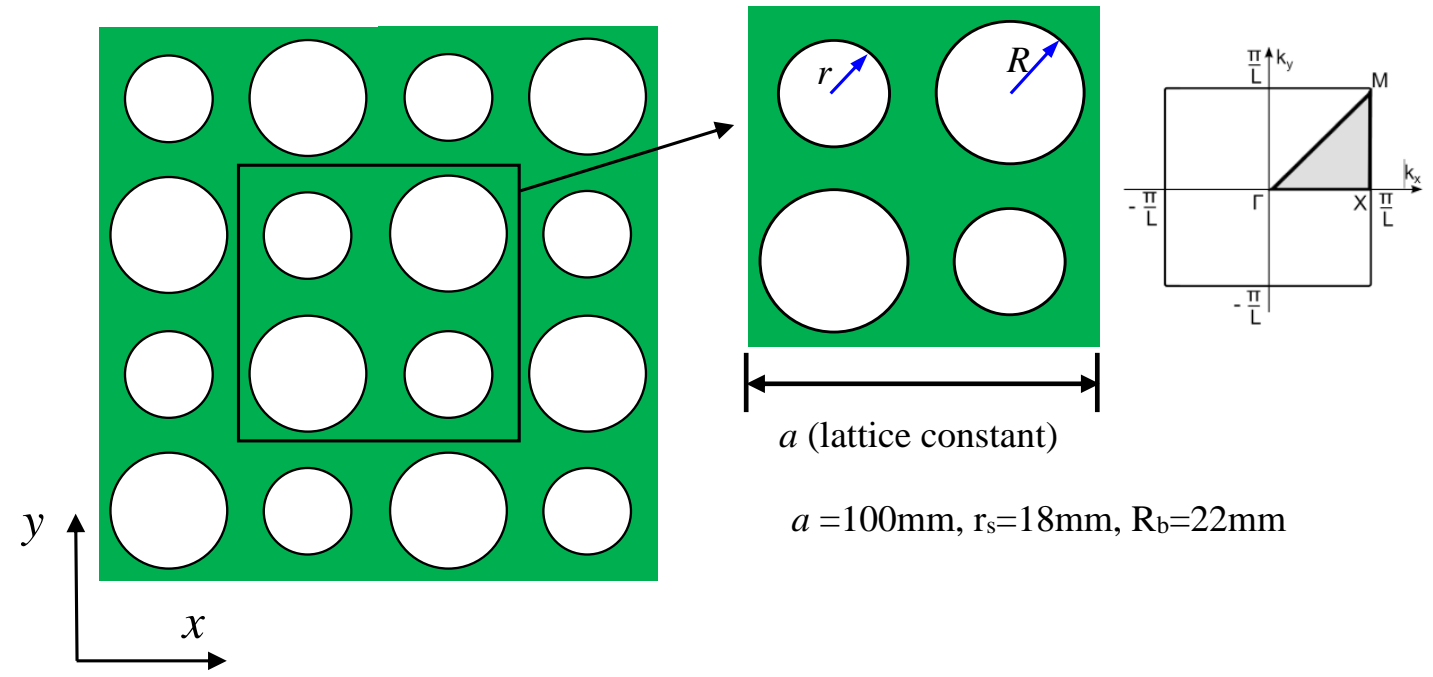

Figure 15: Liquid PCs with different size of hard inclusion

As shown in Fig. 15, the different size of hard inclusion (two big and two small circle hard inclusions) in one unit cell of liquid PCs (lattice constant $a=0.1 \mathrm{~m}$ ) is furthered 
analyzed using the present MR-FEM. The radii for small and big hard inclusions are $r_{\mathrm{s}}=18 \mathrm{~mm}$ and $R_{\mathrm{b}}=22 \mathrm{~mm}$, respectively.

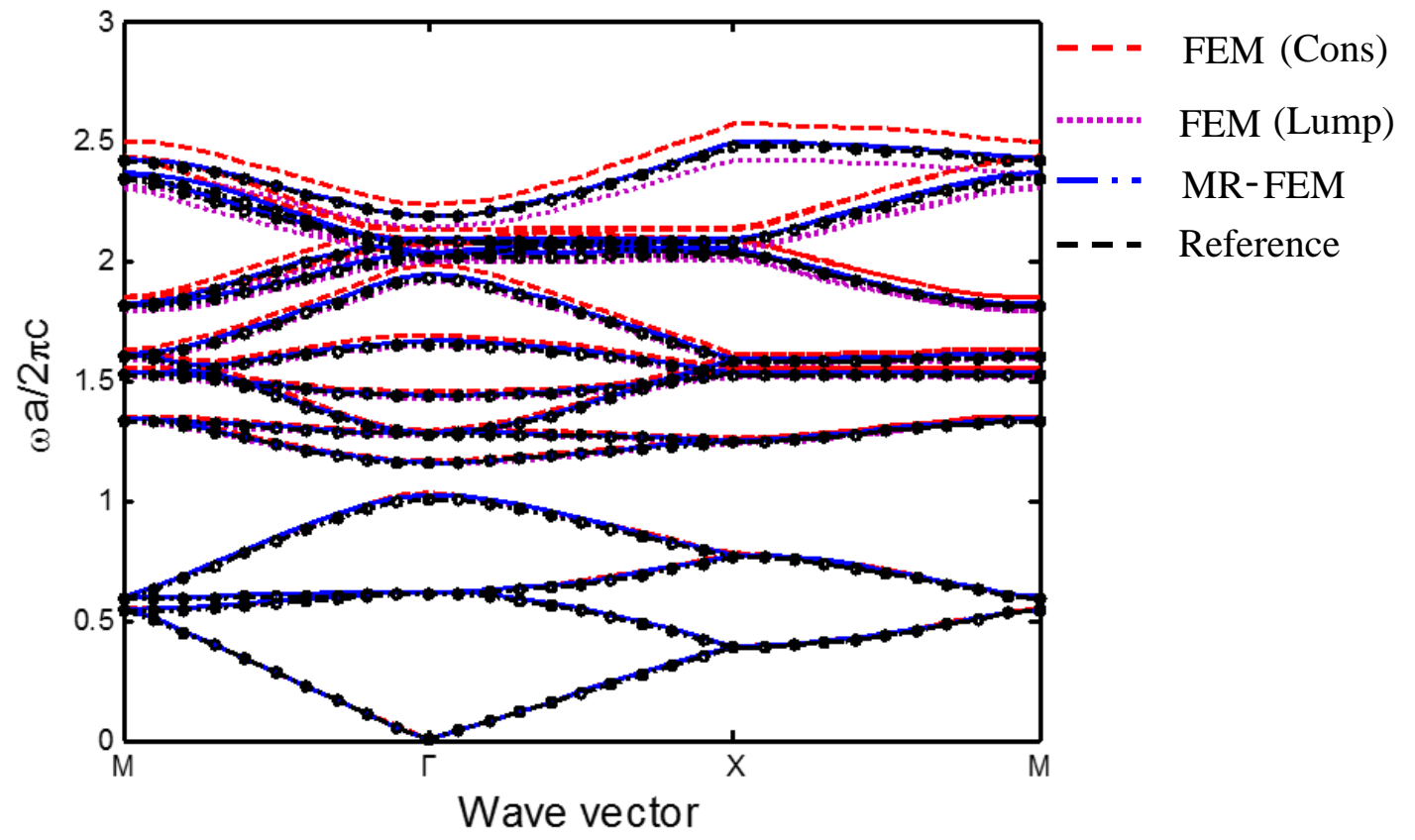

Figure 16: The computed band gap of Liquid PCs with different size of hard inclusion

The band gap computed by standard FEM and MR-FEM using the same coarse mesh (135 nodes, 198 triangular elements) is plotted in Fig. 16. The reference solutions from COMSOL (5378 nodes, 9860 triangular element) are plotted together for the purpose of comparison. In this example, it is clearly indicated that all the numerical solutions agree very well with the reference model. However, a large deviation between the FEM results (lumped and consistent mass) and the reference solutions at high mode shape is clearly observed in Fig. 16. Due to the perfect balance between stiffness and mass in the MRFEM model, the numerical solutions from MR-FEM using coarse mesh always are always very close to the reference results. This example has demonstrated again that the MR-FEM is able to effectively minimize the dispersion error of longitudinal wave in the liquid PCs with hard inclusions. 
In order to further verify the performance of MR-FEM, the pressure contours at the fifteenth model shape (without loss of generality, the fifteen mode shape is randomly selected) are computed. It is clearly noticed in Fig. 17 that the displacement contour from MR-FEM model (117 nodes, 150 linear triangular elements) is in a very good agreement with the reference results obtained from COMSOL (5378 nodes, 9860 high order triangular elements), compared with FEM results using the same type of coarse mesh. This has validated again that the MR-FEM is perfectly appropriate to predict the mode shape of the liquid PCs using coarse mesh.

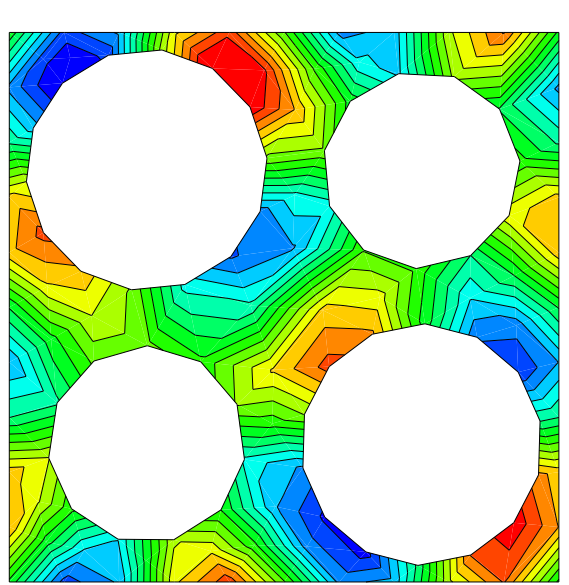

(a) $\operatorname{FEM}(\mathrm{Cons})$

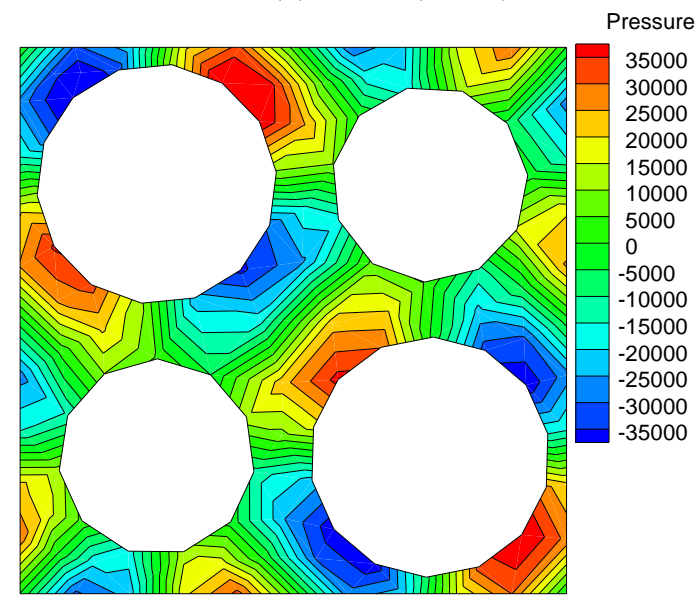

(c) MR-FEM

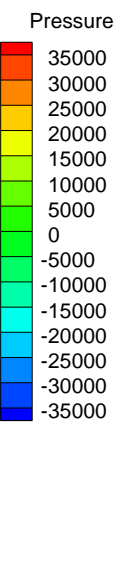

ressure

35000

20000

15000

(5000

0000

5000

$-30000$

5000

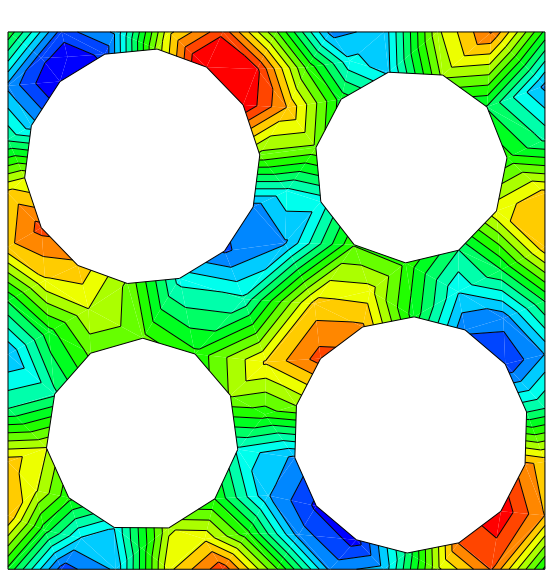

Pressure
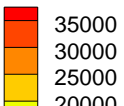

25000
20000

15000

10000

5000

0
-5000

$-5000$

$-10000$

$-15000$

$-25000$

$-30000$

$-35000$

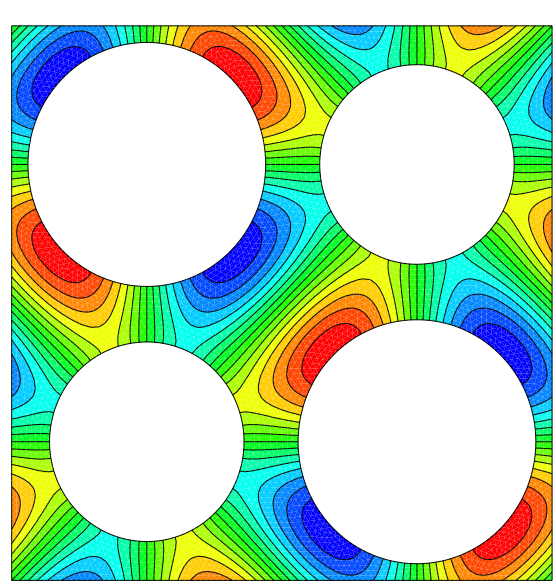

(d) Reference model

Figure 17: The pressure contours at $15^{\text {th }}$ mode shape along $\Gamma$ direction 


\subsection{Liquid PCs with defects}
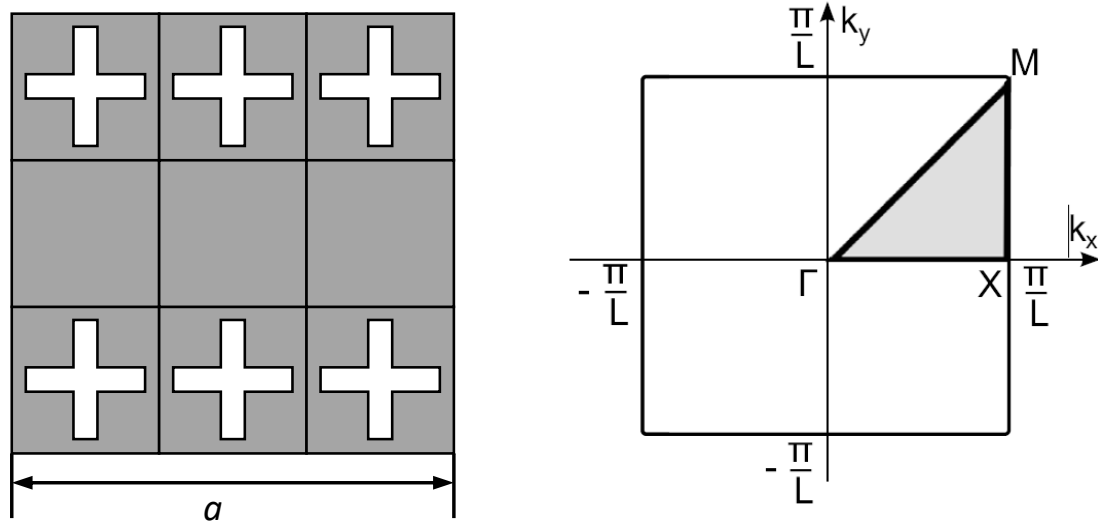

Figure 18: Defects in the liquid PCs with hard inclusion

Recently, the introduction of defects in the liquid PCs is of wide interest as it has many useful advantages such as waveguides and cavities. However, computer simulation of defects in the liquid PCs is more challenging due to extremely expensive computational time. For this purpose, it is necessary to develop more powerful and efficient numerical methods in the design PCs with defects. As shown in Fig. 18, the line defect is introduced in the $3 \times 3$ supercells, and the lattice constant $a=0.15 \mathrm{~m}$. The predictions for the first forty eigenfrequencies (along $\mathrm{M}, \Gamma, \mathrm{X}$ directions) in the liquid PCs with the line defects using coarse mesh (305 nodes, 488 triangular elements) are shown Table 3. For the purpose of comparison, the reference solutions are also obtained from COMSOL software with a set of very fine mesh (64416 nodes and 32048 triangular elements). It is clearly observed that the MR-FEM stands out very clearly compared with FEM using consistent and lumped mass matrices.

Table 3: Numerical errors of eigenfrequency for the first forty eigenfrquencies

\begin{tabular}{cccc}
\hline \hline Direction & FEM(Cons) & FEM(Lump) & MR-FEM \\
$\mathrm{M}$ & $9.18 \%$ & $4.52 \%$ & $3.01 \%$ \\
$\Gamma$ & $9.23 \%$ & $4.43 \%$ & $3.04 \%$ \\
$\mathrm{X}$ & $9.13 \%$ & $4.59 \%$ & $3.11 \%$ \\
\hline \hline
\end{tabular}




\section{Conclusion}

In this work, a mass-redistributed finite element method (MR-FEM) is formulated to analyze the band gap of liquid phononic crystals with hard inclusion. The mass conservation of MR-FEM gives us a great freedom to tune the balance between the stiffness and mass using flexible integration points. The theoretical analysis has indicated that we can even use the integration points outside the triangular elements, which is extremely significant to improve the computational efficiency of traditional FEM model. In addition, the triangular elements which are very easy to capture the complicated geometry are employed in the MR-FEM. Furthermore, the implementation of MR-FEM is very straightforward without increasing computational cost compared with standard FEM. The excellent property of MR-FEM demonstrated in this work has the great potential and capability to deal with large scale problems and nonlinear phononic crystals.

\section{Acknowledgements}

The project is supported by the Project funded by China Postdoctoral Science Foundation. The authors also wish to thank Research Project of the Science Fund of State Key Laboratory of Advanced Design and Manufacturing for Vehicle Body (Grant No. 51375001 and 31615002), Research Project of State Key Laboratory of Mechanical Systems and Vibration (MSV 201613 and MSV201711), and Open Foundation of the State Key Laboratory of Fluid Power and Mechatronic Systems GZK F-201601. 


\section{Appendix}

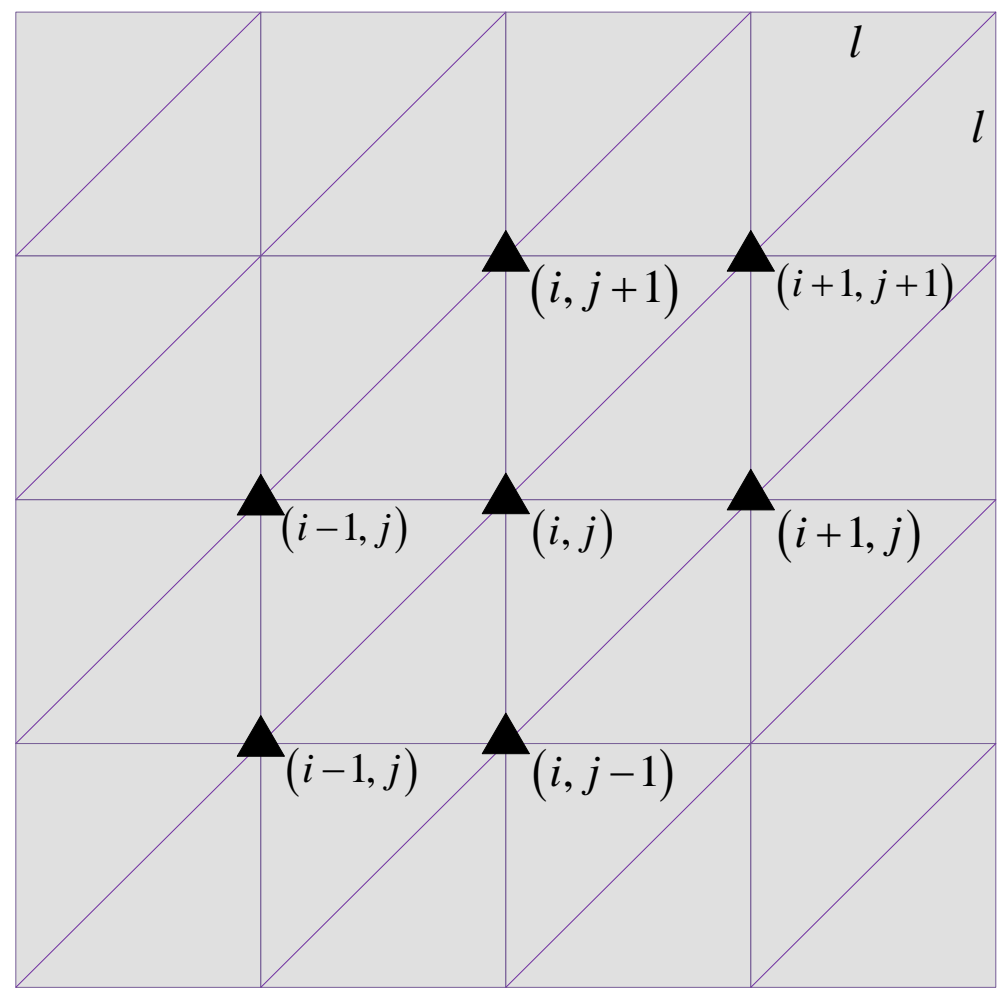

Figure 19: Dispersion error analysis in the framework of MR-FEM

The minimum dispersion error in the propagation of longitudinal wave based on MR-

FEM is derived, which is based on the isotropic media. As depicted in Fig. 19, by using the regular triangular mesh with a constant node spacing $l$, the discretized system of Eq. (4) using MR-FEM is easily formulated. In general, the propagation of longitudinal wave can be expressed in the following form in two-dimensional problems:

$$
p(x)=P_{A} e^{i\left(\kappa_{1} x+\kappa_{2} y\right)}
$$

where $\kappa_{1}=\kappa^{l} \cos \alpha \quad \kappa_{2}=\kappa^{l} \sin \alpha$

The relative dispersion error $e(\alpha, \kappa)$ is defined by the following equation: 


$$
e(\alpha, \kappa)=\left|\frac{\kappa-\kappa^{l}}{\kappa^{l}}\right|
$$

where $\kappa^{l}$ is the approximated wavenumber, and $\kappa$ represents the actual wavenumber. The dispersion error of MR-FEM model in the analysis of the propagation of longitudinal wave is expressed as follows:

$$
e(\alpha, \kappa)=\left|\frac{\left(\kappa^{l}\right)^{2} l^{2}}{24}\left(2 \cos ^{4} \alpha-2 \cos ^{2} \alpha+\left(3 r^{2}-2 r-1\right) \sin 2 \alpha+6 r^{2}-4 r-1\right)+o\left(\left(\kappa^{h}\right)^{4}\right)\right|
$$

As the consistent mass matrix corresponding to $r=2 / 3$ or $r=0$ is used, the following dispersion error is obtained:

$$
e(\alpha, \kappa)=\left|\frac{\kappa^{2} l^{2}}{24}\left(-\frac{1}{2} \sin ^{2} 2 \alpha-\sin 2 \alpha-1\right)+o\left(\kappa^{4}\right)\right|
$$

Thus, the dispersion error for consistent mass is bounded by $\left|-\frac{1}{2} \sin ^{2} 2 \theta-\sin 2 \theta-1\right| \leq \frac{5}{2}$.

As the lumped mass matrix is used (the location parameter of integration point $r=1$ ), the dispersion error can be given in the following equation:

$$
e(\alpha, \kappa)=\left|\frac{\kappa^{2} h^{2}}{24}\left(-\frac{1}{2} \sin ^{2} 2 \alpha+1\right)+o\left(\kappa^{4}\right)\right|
$$

Eq. (A5) indicates that the leading error in the lumped mass is bounded by $\left|-\frac{1}{2} \sin ^{2} 2 \alpha+1\right| \leq 1$

It is obvious that the dispersion error is minimized if the term $6 r^{2}-4 r-1=0$ in Eq. (A3). In that case, the minimum dispersion error is obtained.

$$
e(\alpha, \kappa)=\left|\frac{\kappa^{2} l^{2}}{24}\left(-\frac{1}{2} \sin ^{2} 2 \alpha-\frac{1}{2} \sin 2 \alpha\right)+o\left(\kappa^{4}\right)\right|
$$




\section{Reference}

[1] Olsson RH, El-Kady I. Microfabricated phononic crystal devices and applications. Measurement Science and Technology. 2009;20.

[2] Lin S-CS, Huang TJ. Acoustic mirage in two-dimensional gradient-index phononic crystals. Journal of Applied Physics. 2009;106:053529.

[3] Lin S-CS, Huang TJ, Sun J-H, Wu T-T. Gradient-index phononic crystals. Physical Review B. 2009;79:094302.

[4] Zhu R, Huang GL, Hu GK. Effective Dynamic Properties and Multi-Resonant Design of Acoustic Metamaterials. Journal of Vibration \& Acoustics. 2012;134:319-21.

[5] Huang HH, Sun CT. Wave attenuation mechanism in an acoustic metamaterial with negative effective mass density. New Journal of Physics. 2009;11.

[6] Liu Z, Zhang X, Mao Y, Zhu YY, Yang Z, Chan CT, et al. Locally resonant sonic materials. Science. 2000;289:1734-6.

[7] Wang G, Wen XS, Wen JH, Shao LH, Liu YZ. Two-dimensional locally resonant phononic crystals with binary structures. Physical Review Letters. 2004;93.

[8] Lin S-CS, Huang TJ. Tunable phononic crystals with anisotropic inclusions. Physical Review B. 2011;83:174303.

[9] Hussein MI, Frazier MJ. Band structure of phononic crystals with general damping. Journal of Applied Physics. 2010;108:093506.

[10] Hussein MI, Leamy MJ, Ruzzene M. Dynamics of Phononic Materials and Structures: Historical Origins, Recent Progress, and Future Outlook. Applied Mechanics Reviews. 2014;66.

[11] Khelif A, Adibi A, SpringerLink (Online service). Phononic Crystals Fundamentals and Applications. 1st ed2016. p. 1 online resource (VII, 245 p. 129 illus., 88 illus. in color.).

[12] Xiao SS, Shen LF, He SL. A plane-wave expansion method based on the effective medium theory for calculating the band structure of a two-dimensional photonic crystal. Physics Letters A. 2003;313:132-8.

[13] Shi SY, Chen CH, Prather DW. Plane-wave expansion method for calculating band structure of photonic crystal slabs with perfectly matched layers. Journal of the Optical Society of America a-Optics Image Science and Vision. 2004;21:1769-75. 
[14] Srivastava A, Nemat-Nasser S. Mixed-variational formulation for phononic bandstructure calculation of arbitrary unit cells. Mechanics of Materials. 2014;74:67-75.

[15] Lu Y, Srivastava A. Combining Plane Wave Expansion and Variational Techniques for Fast Phononic Computations. Journal of Engineering Mechanics. 2017:(Accepted).

[16] Wang G, Wen JH, Liu YZ, Wen XS. Lumped-mass method for the study of band structure in two-dimensional phononic crystals. Physical Review B. 2004;69.

[17] Cao Y, Hou Z, Liu Y. Finite difference time domain method for band-structure calculations of two-dimensional phononic crystals. Solid State Communications. 2004;132:539-43.

[18] Liu Y, Gao L-t. Explicit dynamic finite element method for band-structure calculations of 2D phononic crystals. Solid State Communications. 2007;144:89-93.

[19] Zhao JF, Li Y, Liu WK. Predicting band structure of 3D mechanical metamaterials with complex geometry via XFEM. Computational Mechanics. 2015;55:659-72.

[20] Liu GR, Nguyen TT. Smoothed finite element methods. Boca Raton: Taylor \& Francis; 2010.

[21] He ZC, Li GY, Zhong ZH, Cheng AG, Zhang GY, Li E, et al. An ES-FEM for accurate analysis of 3D mid-frequency acoustics using tetrahedron mesh. Computers \& Structures. 2012;106:125-34.

[22] He Z, Li G, Zhang G, Liu G-R, Gu Y, Li E. Acoustic analysis using a massredistributed smoothed finite element method with quadrilateral mesh. Engineering Computations. 2015;32:2292-317.

[23] He ZC, Li E, Li GY, Wu F, Liu GR, Nie X. Acoustic simulation using alpha-FEM with a general approach for reducing dispersion error. Engineering Analysis with Boundary Elements. 2015;61:241-53.

[24] He ZC, Li GY, Liu GR, Cheng AG, Li E. Numerical investigation of ES-FEM with various mass re-distribution for acoustic problems. Applied Acoustics. 2015;89:222-33. [25] Li E, He ZC, Jiang Y, Li B. 3D mass-redistributed finite element method in structural-acoustic interaction problems. Acta Mechanica. 2015:1-23.

[26] Li E, He ZC, Xu X, Liu GR. Hybrid smoothed finite element method for acoustic problems. Computer Methods in Applied Mechanics and Engineering. 2015;283:664-88. 
[27] Liu GR, Dai KY, Nguyen TT. A smoothed finite element method for mechanics problems. Computational Mechanics. 2007;39:859-77.

[28] Liu GR, Nguyen TT, Dai KY, Lam KY. Theoretical aspects of the smoothed finite element method (SFEM). International Journal for Numerical Methods in Engineering. 2007;71:902-30.

[29] Liu GR. A generalized gradient smoothing technique and the smoothed bilinear form for Galerkin formulation of a wide class of computational methods. International Journal of Computational Methods. 2008;5:199-236.

[30] Liu GR, Nguyen TT. Smoothed finite element methods. Boca Raton: Taylor \& Francis,; 2010. p. 691 p.

[31] Nguyen T-K, Nguyen V-H, Chau-Dinh T, Vo TP, Nguyen-Xuan H. Static and vibration analysis of isotropic and functionally graded sandwich plates using an edgebased MITC3 finite elements. Composites Part B: Engineering. 2016;107:162-73.

[32] Li E, Zhang ZP, Chang CC, Liu GR, Li Q. Numerical homogenization for incompressible materials using selective smoothed finite element method. Composite Structures. 2015;123:216-32.

[33] Li E, Zhang ZP, He Z, Xu X, Liu GR, Li Q. Smoothed finite element method with exact solutions in heat transfer problems. International Journal of Heat and Mass Transfer. 2014;78:1219-31.

[34] He ZC, Li E, Wang G, Li GY, Xia Z. Development of an efficient algorithm to analyze the elastic wave in acoustic metamaterials. Acta Mechanica. 2016;227:3015-30.

[35] Li E, He ZC, Wang G. An exact solution to compute the band gap in phononic crystals. Computational Materials Science. 2016;122:72-85.

[36] He ZC, Li E, Liu GR, Li GY, Cheng AG. A mass-redistributed finite element method (MR-FEM) for acoustic problems using triangular mesh. Journal of Computational Physics. 2016;323:149-70.

[37] Li E, He ZC, Jiang Y, Li B. 3D mass-redistributed finite element method in structural-acoustic interaction problems. Acta Mechanica. 2016;227:857-79.

[38] Wang Y-F, Wang Y-S. Multiple wide complete bandgaps of two-dimensional phononic crystal slabs with cross-like holes. Journal of Sound and Vibration. 2013;332:2019-37. 
[39] Liu GR. On Partitions of Unity Property of Nodal Shape Functions: Rigid-BodyMovement Reproduction and Mass Conservation. International Journal of Computational Methods. 2016;13:1640003.

[40] Irimie S, Bouillard P. A residual a posteriori error estimator for the finite element solution of the Helmholtz equation. Computer Methods in Applied Mechanics and Engineering. 2001;190:4027-42.

[41] Ihlenburg F, Babuska I, Sauter S. Reliability of finite element methods for the numerical computation of waves. Advances in Engineering Software. 1997;28:417-24. 